\title{
A Study on Prevalence and Characterization of Candida Species in Immunocompromised Patients
}

\author{
Dhanapal Nandini (D), J. Manonmoney* (D), J. Lavanya, \\ K.V. Leela $(\mathbb{D})$ and Sujith
}

Department of Microbiology, SRM Medical College Hospital and Research Centre, Faculty of Medicine and Health Sciences, SRM Institute of Science and Technology, SRM Nagar, Kattankulathur, Chengalpattu - 603 203, Chennai, TN, India.

\begin{abstract}
Candida spp. is one among the major causes of nosocomial infection, with candidemia gaining increasing prevalence worldwide in parallel with mortality rates ranging from $10-49 \%$. Epidemiology and predisposing factors of candidemia have changed since the number of patients receiving transplants and immunosuppressive therapy, the use of broadspectrum antimicrobials, and the number of AIDS patients have increased. Candidemia is more common among patients with subcutaneous and cutaneous candida infections, through percutaneous inoculation. Major predisposing factors for invasive candidiasis includes neutropenia, haematological malignancies, bone marrow transplantation, total parenteral nutrition, chemotherapy, invasive procedures, and immune-suppressive agents. This study analyses the risk factors of immunocompromised patients with candidemia and antibiogram of Candida spp. isolated from ICU patients. To evaluate the prevalence, distribution and antibiogram of Candida spp., associated risk factors, and outcome in candidemia patients. Blood samples received from patients with clinically suspected fungal infections were subjected to gram staining, culture, sugar assimilation \& fermentation, Candida Chrome agar (CCA) \& Corn meal agar for identification and speciation. Antifungal susceptibility tests were performed by disk-diffusion tests. Among a total of 337 samples received, 22 (6.5\%) samples were positive for candida infections, of which Candida tropicalis $9(41 \%)$ was the predominant isolate followed by C. albicans $5(23 \%)$, Candida glabrata 4 (18\%), Candida parapsilosis 2 (9\%), and Candida krusei 2 (9\%). Male patients had a higher prevalence of candidemia 15 (68.2\%). Among the age group of 51-70 years, uncontrolled DM(Diabetes mellitus) and CKD(chronic kidney disease) were found to be the predominant co-morbidities with candidemia.

Keywords: Invasive fungal infection, candidemia, immunocompromised patients, Candida albicans, Candida nonalbicans, AFST
\end{abstract}

*Correspondence: manonmoj@srmist.edu.in

(Received: October 09, 2020; accepted: September 01, 2021)

Citation: Nandini D, Manonmoney J, Lavanya J, Leela KV, Sujith. A Study on Prevalence and Characterization of Candida Species in Immunocompromised Patients. J Pure Appl Microbiol. 2021;15(4):2065-2072. doi: 10.22207/JPAM.15.4.29

(C) The Author(s) 2021. Open Access. This article is distributed under the terms of the Creative Commons Attribution 4.0 International License which permits unrestricted use, sharing, distribution, and reproduction in any medium, provided you give appropriate credit to the original author(s) and the source, provide a link to the Creative Commons license, and indicate if changes were made. 


\section{INTRODUCTION}

Invasive fungal infections are associated with significant health problems, especially nosocomial infections. Among which one-fourth of blood-stream infections (BSI) are caused by Candida species. Candida non-albicans have immensely replaced Candida albicans in majority of candida infections, although the distribution of species varies with the geographical region and patient population. ${ }^{1-3}$

Candida tropicalis has been found to be the major fungal isolate from blood stream infection/candidemia in India and Taiwan, whereas Candida parapsilosis and Candida glabrata have been found to be prominent in China and the US respectively. ${ }^{2,4}$ Candida has been reported as the fourth to sixth common species isolated from nosocomial infections in the USA and Switzerland. ${ }^{2,5,6}$ Candida spp. comprise $96 \%$ of all opportunistic infections and is also one among the top reasons for sepsis, accounting for $9 \%$ of the total pathogenic microbes., ${ }^{7,8}$

Invasive candidiasis has been increasingly associated with prolonged hospital stays usage of in-dwelling devices, critically ill patients with underlying diseases, and immunocompromised patients. Mortality rates among these patients are high due to candidemia (30-81\% cases) and delayed specific antifungal therapy. ${ }^{1,5}$ Approximately $3355 \%$ of all candidemia reports arise from intensive care units (ICUs). ${ }^{5}$ The portal of entry in majority of cases is the gut, where they thrive and can break the intestinal layers to enter bloodstream, other portals include skin, in patients with indwelling catheters (central venous catheters; CVCs). ${ }^{7,9}$

One of the major underlying comorbidities of patients with candidemia is malignancy and diabetes mellitus, Malignancy patients with candidemia have a mortality rate of $30-50 \%$ due to the increasing resistance in isolates 2,4 . Azole drug resistance in India among Candida species has been increasingly been observed, with Fluconazole resistance ranging from $4.9 \%$ to $37.5 \%$, itraconazole resistance ranging from 2.9 to $24 \%$ and voriconazole resistance $56 \% .{ }^{10}$ Some of the independent risk factors of cardinal importance, associated with candidemia include cancer chemotherapy, neutropenia, total parental nutrition administration, use of broadspectrum antimicrobials, indwelling vascular catheters, and mucosal colonisation by Candida spp. ${ }^{4,9,10}$

Understanding the recent trends in blood stream infections, the specific etiological agents, antibiogram, epidemiology and risk factors is very crucial to select the appropriate treatment modalities and reduce the number of mortality rates in affected patients. This study aims to find the prevalence, distribution and risk factors related to isolation of Candida species from blood samples of in-patients from a tertiary care centre.

Aim

To determine the prevalence of candidemia and its associated risk factors and outcome in candidemic patients, as well as to speciate and study the resistance pattern of the isolated Candida spp.

\section{MATERIALS AND METHODS}

Observational and descriptive study was conducted in the Department of Microbiology, SRM Medical College Hospital and Research Centre (SRM MCH \& RC), Potheri, for a period of 1year from January 2019- January 2020. ICU (Intensive Care Unit).

Patients of all age groups and gender, suspected of invasive fungal infections, attending SRM MCH \& RC were included in the study. Superficial fungal infections, patients on antifungal therapy, and patients diagnosed with bacterial infections were excluded. Blood samples were collected under aseptic precautions as per standard protocol. (CDC guidelines)

Blood cultures were loaded into automated system BACT/ALERT 3D as per manufacturer's protocol.

All positive samples were processed by gram stain, followed by culture with Sabouraud dextrose agar(SDA; with Chloramphenicol \& Cycloheximide) and incubated at $25^{\circ} \mathrm{C}$ and $37^{\circ} \mathrm{C}$. Identification and speciation of isolates were performed by analysis of growth on corn meal agar (for chlamydospore production) and Candida chrome agar; CCA (for species differentiation), along with germ tube test, and sugar assimilation and sugar fermentation tests (glucose, maltose, sucrose, lactose, cellulose, galactose, trehalose, xylose, dulcitol). Antifungal susceptibility testing was performed on Mueller-Hinton agar (MHA) using Amphotericin-B, Fluconazole, Voriconazole, 
and Itraconazole discs, according to CLSI guidelines (M44-A) by Kirby-Bauer disc diffusion method and incubated at $35^{\circ} \mathrm{C}$ for $24-48$ hours.

\section{RESULT}

A sum total of 337 blood samples were received from ICU patients, among which 22 (6.5\%) were positive and $93.5 \%$ negative for isolation of Candida spp. Out of the 22 samples (total positive), 15 (68.2\%) were men and 7 (31.8\%) were women. Immunocompromised patients comprised $100 \%$ of the total positive cases (Fig. 1). Candidemia was found more commonly in the age group of 51-60 years (Table 1). Patients with diabetes mellitus (DM) and chronic kidney disease on dialysis, were more susceptible to candidemia among the immunocompromised patients (Table. 2). Risk factors among immunocompromised patients admitted in the ICU whose blood cultures were

Table 1. Age distribution in study population

\begin{tabular}{lc}
\hline $\begin{array}{l}\text { Age } \\
\text { Group }\end{array}$ & $\begin{array}{c}\text { Number of } \\
\text { Patients (\%) }\end{array}$ \\
\hline$<10$ & 0 \\
$11-20$ & $1(4.0 \%)$ \\
$21-30$ & $1(4.0 \%)$ \\
$31-40$ & $2(9.0 \%)$ \\
$41-50$ & $3(13.0 \%)$ \\
$51-60$ & $8(36.0 \%)$ \\
$61-70$ & $5(21.0 \%)$ \\
$71-80$ & $2(9.0 \%)$ \\
$81-90$ & $1(4.0 \%)$ \\
Total & 22 \\
\hline
\end{tabular}

received in microbiology laboratory are tabulated Table 3, in which Diabetes mellitus (DM) was found to be predominant (24.9\%), closely followed by Chronic kidney disease(CKD; 20\%).

In the study period, 22 isolates of Candida species were identified from patients (Fig. 2), out of which Candida tropicalis was the most predominant isolate, 9 (41\%), followed by C. albicans, 5 (23\%), Candida glabrata, 4 (18\%), Candida parapsilosis, 2 (9\%), and Candida krusei, 2 (9\%).

Antifungal susceptibility test performed by disk diffusion method revealed resistance to Amphotericin B in Candida tropicalis (22.2\%), Candida albicans (20\%) and Candida glabrata (25\%). Intermediate sensitivity to Amphotericin B was found in Candida tropicalis (11.1\%) and Candida glabrata (25\%) (Table 4). Susceptibledose dependent to intrinsic resistant phenotypes were observed to Fluconazole in Candida glabrata

Table 2. Distribution of age and gender among immunocompromised patients $(n=22)$

\begin{tabular}{lcccc}
\hline \multirow{2}{*}{$\begin{array}{l}\text { Age } \\
\text { Group }\end{array}$} & $\begin{array}{c}\text { Immuno- } \\
\text { compromising } \\
\text { Conditions }\end{array}$ & \multicolumn{3}{c}{ Gender } \\
\cline { 3 - 5 } & & Males & Females & Total \\
\hline $31-40$ & Leukaemia & 3 & 1 & 4 \\
$51-60$ & Diabetes mellitus & 6 & 3 & 9 \\
$61-70$ & $\begin{array}{c}\text { Chronic kidney disease } \\
\text { (on Dialysis) }\end{array}$ & 4 & 3 & 7 \\
$61-70$ & AIDS & 2 & 0 & 2 \\
Total & & 15 & 7 & 22 \\
\hline
\end{tabular}

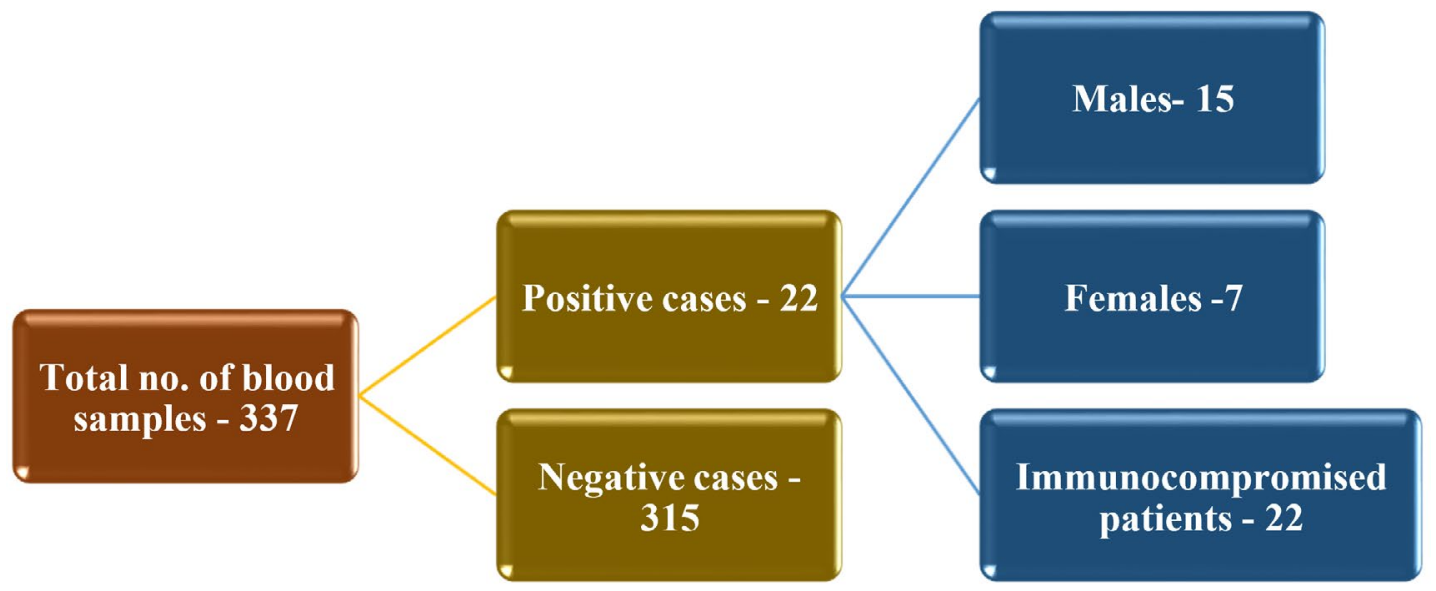

Fig. 1. Distribution of positive cases in the study population. 
(100\%), and Candida krusei (100\%). All isolates were found to be sensitive to Itraconazole and Voriconazole.

\section{DISCUSSION}

Candidemia is known to cause increased rates of morbidity and mortality worldwide, particularly in immunocompromised patients. Predominant species causing candidemia has changed from Candida albicans to Candida nonalbicans species in recent years. Candida tropicalis and Candida parapsilosis are the commonly found isolates in candidemia in Southern India. ${ }^{8}$

Table 3. Incidence of risk factors in immunocompromised patients admitted in ICU

\begin{tabular}{lll}
\hline No. & $\begin{array}{l}\text { Risk Factors In Immuno- } \\
\text { compromised Patients }\end{array}$ & Number (\%) \\
\hline 1. & Diabetes mellitus & $83.57(24.9)$ \\
2. & Chronic Kidney disease(CKD) & $67.4(20)$ \\
3. & Malignancy (Lymphoma/ & $60.7(18)$ \\
& tumor/ leukaemia) & \\
4. & Congestive heart failure & $33(9.9)$ \\
5. & Myocardial infarction & $32(9.4)$ \\
6. & Vascular disease & $25.2(7.4)$ \\
7. & Chronic liver disease & $12.7(3.9)$ \\
8. & Chronic respiratory illness & $10.7(3.2)$ \\
9. & Connective tissue disease & $5(1.4)$ \\
10. & AlDS & $3.7(1)$ \\
11. & Chronic neurological disorder & $3(0.8)$ \\
& Total & $337(100 \%)$ \\
\hline
\end{tabular}

Malignancy and diabetes mellitus have been reported as the major comorbidities of patients with candidemia. The major risk factors that can increase the susceptibility to candidemia includes, increased use of corticosteroids and antibiotics, prolonged hospital stays, neutropenia, cancer chemotherapy, AIDS, intravascular catheterization, and other immunosuppressing conditions. Candida infections are becoming more prevalent in intensive care units and critical care units other than immunocompromised patients.

Candidemia was found to be $6.5 \%$ in this study, among 337 cases, included in the study. Gadham NR et al. ${ }^{1}$ and Thomas $M$ et al. ${ }^{8}$ reported a prevalence of $14.8 \%$ among 225 samples and $7.3 \%$ among 1440 samples respectively, while Tak $V$ et al. ${ }^{5}$ reported a prevalence of $74 \%$ in a period of 4 years.

A higher prevalence of candidemia in men (68.2\%) was observed compared to women $(31.8 \%)$ in our study population, (M:F ratio $=1.8)$. Similar results were observed in a comparative study performed by Dimopoulus $\mathrm{G}$ et al. ${ }^{9}$, with higher prevalence of candidemia in men in both immunocompromised $(\mathrm{M}: \mathrm{F}=1.2)$ and non-immunocompromised patients ( $\mathrm{M}: \mathrm{F}=1.5$ ) compared to women. Alkharashi $\mathrm{N}$ et al. ${ }^{6}$ had reported in their 10-year study on candidemia that $53.4 \%$ men were affected compared to $46.6 \%$ women ( $\mathrm{M}: \mathrm{F}=1.14)$, within the mean age group 49.7 \pm 28.1 . The most predominantly affected age groups were 5160 years (36.3\%), closely followed by $61-70$ years $(22.7 \%)$ in our study.

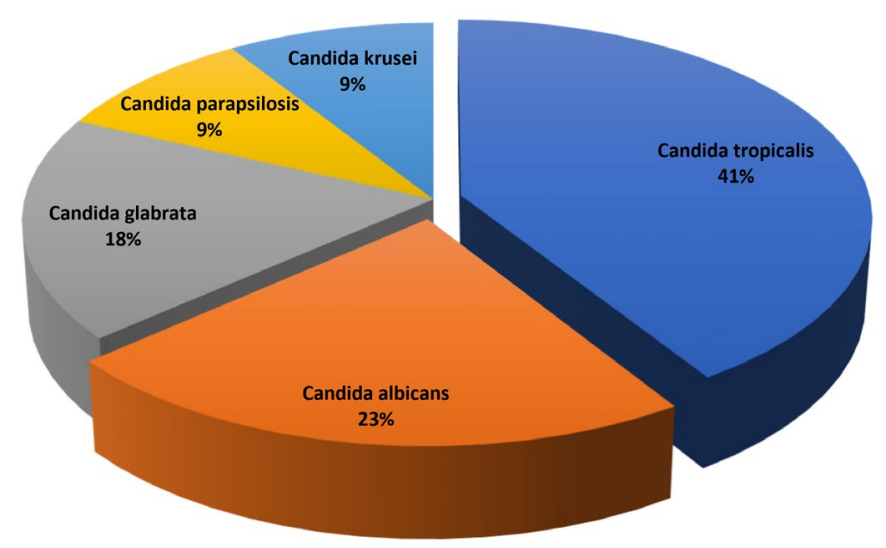

- Candida tropicalis = Candidaalbicans $=$ Candida glabrata

Fig. 2. Distribution of Candida species among (immunocompromised) patients $(n=22)$. 


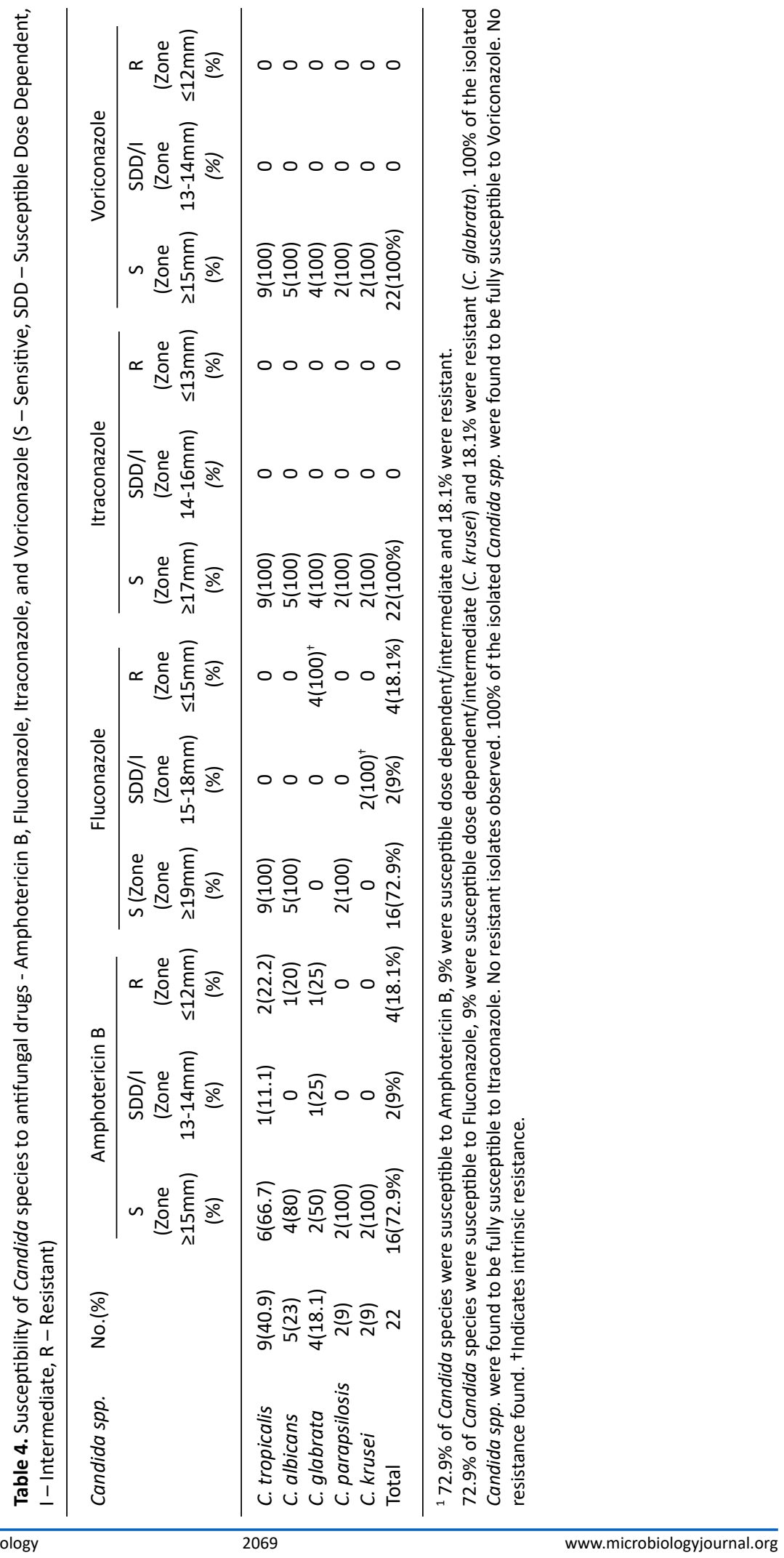


Among immunocompromised patients in our study, 51-60 years age group had the highest number of candidemia patients, with diabetes mellites as a co-morbidity, followed by patients with chronic kidney disease undergoing dialysis, Leukaemia, and HIV. A univariate regression analysis study by Michalopuolos et al. confirmed that DM is factor significantly associated with 30day mortality in candidemia, and is a risk factor in more than $50 \%$ cases. ${ }^{11}$

In a study on candidemia in haemodialysis patients by Pyrgos $\mathrm{V}$ et al. ${ }^{12}, 22 \%$ among the total candidemia patients were found to be adult haemodialysis patients, among which antibiotic use and implantable vascular catheter were recorded contribute as major risk factors. DM followed by HIV were the most predominant risk factors among immunocompromised patients with candidemia in a study by Dutta BS et al. ${ }^{13}$

Gastrointestinal carcinoma (46.2\%) was reported as the most common malignancy in candidemia patients along with other underlying co-morbidities such as DM (30.9\%) in studies by Wu PF et al. ${ }^{2}$ and A. Chakrabarti et al. ${ }^{10}$, while in our study leukaemia (14.7\%) was reported as the most common malignancy among immunocompromised patients. Chakrabarti A et al. ${ }^{14}$ along with other studies ${ }^{4,15}$ reported pneumonia (32.9\%), acute renal failure $(61.2 \%)$, and long-term use of antibiotics (64.1\%) as the common underlying co-morbidities among ICU patients with candidemia.

Candida species isolated from blood on repeat samples were considered as a pathogen in this study. Candida tropicalis was found to be the dominant isolate (41\%), followed by Candida albicans (23\%) among the total study population $(n=22)$. Similar trends of Candida non-albicans have been isolated and observed in various studies, ${ }^{1,5,7,8,16-19}$ while other studies show a predominance of Candida glabrata and Candida albicans. ${ }^{20-23}$ Phospholipase activity and biofilm formation have been found to be important virulence factors in $\mathrm{C}$. tropicalis contributing to pathogenicity in candidemia patients. ${ }^{19,24}$

Regarding antifungal susceptibility testing, most of the isolates were found to be sensitive to all the four drugs tested including Amphotericin B (72.7\%), Fluconazole (81.8\%),
Itraconazole (100\%) and Voriconazole (100\%) similar to other studies. ${ }^{20,16,22,25}$ Candida glabrata was however found to be fully resistant (100\%) because of intrinsic resistance to Fluconazole alone. ${ }^{16,20}$ Few isolates showed resistance (18.1\%) and intermediate sensitivity (3.6\%) to Amphotericin B. Yesudhason. B et al. ${ }^{26}$ reveals the resistance pattern of $\mathrm{C}$. tropicalis to Itraconazole (26.2\%), Ketoconazole (24.6\%) and Fluconazole (37.7\%).

The changing trends of epidemiology and etiological agents causing invasive fungal infections among the increasing number of immunocompromised group of patients are posing a significant challenge in management and treatment of the disease. ${ }^{27}$ Increasing resistance among isolates despite the current antifungal agents have led to investigations to find more rapid detection techniques and treatment modalities. Prompt and appropriate identification of fungal species and following stringent antifungal stewardship may aid in management of the diseases and reduce mortality rates. ${ }^{27-30}$

\section{CONCLUSION}

The present study was performed on blood samples with majority of patients being males belonging to the age group of 51-70 years. Among immunocompromised patients, uncontrolled diabetes mellitus and chronic kidney disease were found to increase the incidence of candida infections. Through this study we have observed that early detection of etiological agents by microscopy and culture and prompt initiation of antifungal therapy can aid in reduction of morbidity and mortality among immunocompromised patients.

\section{ACKNOWLEDGMENTS}

None.

\section{CONFLICTS OF INTEREST}

The authors declare that there is no conflict of interest.

\section{AUTHORS' CONTRIBUTION}

All the authors listed have made a substantial, direct, and intellectual contribution to the work, and approved it for publication. 


\section{FUNDING}

None.

\section{DATA AVAILABILITY}

All datasets generated or analysed during this study are included in the manuscript.

\section{ETHICS STATEMENT}

This study is approved from the Institute's Ethical Committee (1518/IEC/2018) SRM Medical College Hospital and Research Centre, Chennai, TN, India.

\section{REFERENCES}

1. Gandham NR, Vyawahare CR, Jadhav SV, Misra RN. Candidemia: Speciation and antifungal susceptibility testing from a tertiary care hospital in Maharashtra, India. Medical Journal of Dr. DY Patil University. 2016;9(5):596-599. doi: 10.4103/0975-2870.192165

2. Wu PF, Liu WL, Hsieh MH, et al. Epidemiology and antifungal susceptibility of candidemia isolates of non-albicans Candida species from cancer patients: Non-albicans candidemia in cancer patients. Emerg Microbes Infect. 2017;6(1):1-7. doi: 10.1038/ emi.2017.74

3. Gill M, Sharma S, Khanna A. Emergence of non albicans Candida in a tertiary care hospital of north India. Indian J Microbiol Res. 2018;5(2):244-248 doi: 10.18231/2394-5478.2018.0051

4. Giri S, Kindo AJ, Kalyani J. Candidemia in intensive care unit patients: A one year study from a tertiary care center in South India. J Postgraduate Med. 2013;59(3):190-195. doi: 10.4103/0022-3859.118036

5. Tak V, Mathur P, Varghese P, Gunjiyal J, Xess I, Misra MC. The epidemiological profile of candidemia at an Indian trauma care center. J Lab Physicians. 2014;6(2):96-101. doi: 10.4103/0974-2727.141506

6. Alkharashi N, Aljohani S, Layqah L, et al. Candida Bloodstream Infection: Changing Pattern of Occurrence and Antifungal Susceptibility over 10 Years in a Tertiary Care Saudi Hospital. Can J Infect Dis Med Microbiol. 2019;2019:2015692. doi: 10.1155/2019/2015692

7. Phu TT, Truong NN, Phuong NT, Bup PH, Thao PT. Antifungal Resistance of Candida Spp. in Bloodstream Infection. Biomed J Sci Tech Res. 2020;24(2):1815318155. doi: 10.26717/BJSTR.2020.24.004032

8. Thomas M, Oberoi A, Dewan E. Species distribution and antifungal susceptibility of candidemia at a multispecialty center in North India. CHRISMED J Health Res. 2016;3(1):33-36. doi: 10.4103/23483334.172395

9. Dimopoulos G, Karabinis A, Samonis G, Falagas ME. Candidemia in immunocompromised and immunocompetent critically ill patients: a prospective comparative study. Eur J Clin Microbiol Infect Dis. 2007;26(6):377-384. doi: 10.1007/s10096-007-0316-2

10. Giri S, Kindo AJ. A review of Candida species causing blood stream infection. Indian J Med Microbiol. 2012;30(3):270-278. doi: 10.4103/0255-0857.99484
11. Rodrigues CF, Rodrigues ME, Henriques M. Candida $s p$. infections in patients with diabetes mellitus. J Clin Med. 2019;8(1):76. doi: 10.3390/jcm8010076

12. Pyrgos V, Ratanavanich K, Donegan N, Veis J, Walsh TJ, Shoham S. Candida bloodstream infections in hemodialysis recipients. Medical Mycology. 2009;47(5):463-467. doi : $10.1080 / 13693780802369332$

13. Dutta BS, Sharma A, Hazarika NK, Barua P, Begum S. Distribution of Candida Species amongst Various Clinical Samples from Immunocompromised Patients attending Tertiary Care Hospital, Assam. Int J Curr Microbiol App Sci. 2018;7(8):1004-1019. doi: 10.20546/ijcmas.2018.708.113

14. Chakrabarti A, Sood P, Rudramurthy SM, et al. Incidence, characteristics and outcome of ICUacquired candidemia in India. Intensive Care Med. 2015;41(2):285-295. doi: 10.1007/s00134-014-3603-2

15. Xess I, Jain N, Hasan F, Mandal P, Banerjee U. Epidemiology of candidemia in a tertiary care centre of north India: 5-year study. Infection. 2007;35(4):256259. doi: 10.1007/s15010-007-6144-6

16. Gade N, Neral A, Niza Monga D, et al. Antifungal Susceptibility Pattern of Clinical Isolates of Candida from a Tertiary Care Hospital in Chhattisgarh, India. Saudi J Pathol Microbiol. 2019;4(12):906-913 doi: 10.36348/sjpm.2019.v04i12.007

17. Alagiri SB, Vijayaraman RS, Ramaraj V, Kindo AJ. Invasive yeast infections in the intensive care unit of a tertiary care centre in South India. J Acad Clin Microbiol. 2017;19(1):19-26. doi: 10.4103/jacm. jacm_29_16

18. Rajeevan S, Thomas M, Appalaraju B. Characterisation and antifungal susceptibility pattern of Candida species isolated from various clinical samples at a tertiary care Centre in South India. Indian J Microbiol Res. 2016;3(1):53-57. doi: 10.5958/23945478.2016.00014.5

19. Tulasidas S, Rao P, Bhat S, Manipura R. A study on biofilm production and antifungal drug resistance among Candida species from vulvovaginal and bloodstream infections. Infect Drug Resist. 2018;11:2443-2448. doi: 10.2147/IDR.S179462

20. Kumudhavalli KS. A Study on Invasive Fungal Infections among Immunocompromised Patients in a Tertiary Care Hospital (Doctoral dissertation) Madras Medical College, Chennai: TN MGR Medical University; 2013.

21. Kothalawala M, Jayaweera JA, Arunan S, Jayathilake A. The emergence of nonalbicans candidemia and evaluation of HiChrome Candida differential agar and VITEK2 YST ${ }^{\circledR}$ platform for differentiation of Candida bloodstream isolates in teaching hospital Kandy, Sri Lanka. BMC Microbiol. 2019;19(1):136. doi: 10.1186/ s12866-019-1518-3

22. Tan TY, Hsu LY, Alejandria MM, et al. Antifungal susceptibility of invasive Candida bloodstream isolates from the Asia-Pacific region. Medical Mycology. 2016;54(5):471-477.

23. Ghrenassia E, Mokart D, Mayaux J, et al. Candidemia in critically ill immunocompromised patients: report of a retrospective multicenter cohort study. Ann Intensive Care. 2019;9(1):62. doi: 10.1186/s13613-019-0539-2 
24. Deorukhkar SC, Saini S, Mathew S. Virulence factors contributing to pathogenicity of Candida tropicalis and its antifungal susceptibility profile. Int $J$ Microbiol. 2014;2014:456878. doi: 10.1155/2014/456878

25. Sabhapandit D, Lyngdoh WV, Bora I, Prasad A, Debnath K, Elantamilan D. Prevalence of non-albicans candidemia in a tertiary care hospital in Northeast India. Int J Med Sci Public Health. 2017;6(11):16201625. doi: 10.5455/ijmsph.2017.0823622092017

26. Yesudhason BL, Mohanra MK. Candida tropicalis as a predominant isolate from clinical specimens and its antifungal susceptibility pattern in a tertiary care hospital in Southern India. J Clin Diagn Res. 2015;9(7):DC14-16. doi: 10.7860/ JCDR/2015/13460.6208
27. Low $\mathrm{CY}$, Rotstein C. Emerging fungal infections in immunocompromised patients. F1000 Medicine Reports. 2011;3:14. doi: 10.3410/M3-14

28. Pandey N, Gupta MK, Paul P, Tilak R. Necessity to identify candida species accurately with minimum inhibitory concentration determination in each case of bloodstream infections. Journal of Infection and Public Health. 2020;13(5):753-858. doi: 10.1016/j. jiph.2019.12.002

29. Barnes RA. Early diagnosis of fungal infection in immunocompromised patients. J Antimicrob Chemother. 2008;61(suppl_1):i3-i6. doi: 10.1093/jac/ dkm424

30. Kaur R, Jaggi S, Dhakad MS, Rawat D. An etiological and antifungal profile of candidemia in children. Int $J$ Community Med Public Health. 2019;6(9):3899-3904. doi: 10.18203/2394-6040.ijcmph20193990 